\title{
Construction of relapse-related IncRNA-mediated ceRNA networks in Hodgkin lymphoma
}

\author{
Yuexiong Liang ${ }^{1}$, Haifeng Zhu ${ }^{1}$, Jing Chen ${ }^{1}$, Wei Linn', Bing Li ${ }^{1}$, Yusheng Guo ${ }^{2}$
}

${ }^{1}$ The First People's Hospital of Zhaoging City, Zhaoging, Guangdong Province, China ${ }^{2}$ North Huashan Hospital, Fudan University, Shanghai, China

Submitted: 17 November 2018

Accepted: 2 February 2019

Arch Med Sci 2020; 16 (6): 1411-1418

DOI: https://doi.org/10.5114/aoms.2020.98839

Copyright $\odot 2020$ Termedia \& Banach

\section{Abstract}

Introduction: Hodgkin lymphoma $(\mathrm{HL})$ is a type of lymphoma common throughout the western countries. However, the detailed mechanisms and special biomarkers of $\mathrm{HL}$ remain to be further investigated. Emerging studies have shown that long non-coding RNAs play a key role in human cancers. Material and methods: In the present work, we constructed relapse-related IncRNA-mediated ceRNA networks in HL. Additionally, we constructed co-expression networks for these relapse-related IncRNAs. We also constructed a relapse-related InCRNA-miRNA-mRNA network to study the potential mechanism of these IncRNAs. Furthermore, gene ontology (GO) and Kyoto Encyclopedia of Genes and Genomes (KEGG) pathway analysis were performed to explore functions of DEGs in Hodgkin lymphoma.

Results: A total of 18 IncRNAs were found to be dysregulated between early relapse and late relapse HL. Six IncRNAs (PCBP1-AS1, HCG18, GAS5, PSMD6-AS2, PRKCQ-AS1, SNHG6), 116 mRNAs and 121 miRNAs were included in the ceRNA network. Bioinformatics analyses revealed that these IncRNAs were significantly involved in regulating immune system processes, responses to chemical stimuli and responses to stress. Among them, HCG18 and PCBP1-AS1 were identified as key IncRNAs in HL relapse.

Conclusions: Our results for the first time constructed the key relapse-related IncRNA-mediated ceRNA networks in Hodgkin lymphoma progression. We trust that this work will provide a new therapeutic and prognostic target for HL.

Key words: long non-coding RNA, diffuse large B-cell lymphoma, ceRNA networks, expression profiling, follicular lymphoma.

\section{Introduction}

Hodgkin lymphoma $(\mathrm{HL})$, first described by Thomas Hodgkin, is a lymphoma that represents about $1 \%$ of all de novo neoplasms occurring every year worldwide [1]. According to the World Health Organization classification, $\mathrm{HL}$ comprises 2 types of $\mathrm{HL}$, classical Hodgkin lymphoma (accounting for $95 \%$ of all $\mathrm{HL}$ cases) and nodular lymphocyte predominant $\mathrm{HL}$ (accounting for $5 \%$ of all $\mathrm{HL}$ cases) $[2,3]$. The incidence of $\mathrm{HL}$ was found to be increased in teenagers and patients older than 55 years [4]. However, the mechanisms underlying $\mathrm{HL}$ initiation and progression remained unclear.

Long non-coding RNAs (IncRNAs), a kind of ncRNAs over 200 bps in length, have been found to play key roles in cancer progression by regulating gene expression through transcriptional and post-tran-

\author{
Corresponding author: \\ Yuexiong Liang \\ The First People's Hospital \\ of Zhaoqing City \\ 9 East Donggang Road \\ 526000 Zhaoqing \\ Guangdong Province, China \\ Phone: +86 0758-2102161 \\ Fax: +86 0758-2102161 \\ E-mail: liangyuexiong@sina. \\ com
}


scriptional mechanisms [5-7]. For example, InCRNA EPIC1 promoted breast tumorigenesis by interacting with MYC to induce cell-cycle-related gene expression [8]. MALAT1 interacted with the PRC2 complex to regulate inflammatory gene expression in diabetes [9]. However, the roles of IncRNAs in $\mathrm{HL}$ remained unknown. Until now, only two reports have indicated that IncRNAs were involved in HL progression. Tayari et al. [10] identified HL-specific IncRNAs by using microarray profiling. Leucci et al. [11] also found that MALAT1 could bind to miR-9 to regulate its targets expression in the $\mathrm{cHL}$ cell line $\mathrm{L}-428$. Exploring the potential functions of IncRNAs in $\mathrm{HL}$ could provide useful information to identify novel biomarkers for HL.

In this study, we focused on identifying relapse-related IncRNAs in HL by analyzing the Gene Expression Omnibus (GEO) dataset GSE17920. Next, we conducted co-expression network analysis and bioinformatics analysis for relapse-related IncRNAs in HL. Moreover, we constructed a relapse-related IncRNA-miRNA-mRNA network to explore the potential mechanisms of these IncRNAs. We trust that the work will provide potential new therapeutic and prognostic targets for $\mathrm{HL}$

\section{Material and methods}

\section{LncRNA classification pipeline}

In this work, a pipeline was utilized to identify IncRNA expression pattern in $\mathrm{HL}$, which was described by Zhang et al. [12].

\section{Microarray data and data preprocessing}

In the work, we analyzed GSE17920 to identify relapse-related mRNAs and IncRNAs in Hodgkin lymphoma by downloading GEO datasets. A total of 9 early relapse $\mathrm{HL}$ and 24 late relapse $\mathrm{HL}$ samples were included in GSE17920, which was reported by Christian et al. All samples were obtained from Hodgkin's lymphoma patients.

\section{Functional group analysis}

Here, we performed Gene Ontology (GO) analysis and Kyoto Encyclopedia of Genes and Genomes (KEGG) analysis to predict the potential roles of genes by using the DAVID system (https:// david.ncifcrf.gov/).

\section{Identification of IncRNA-associated PPI modules}

We analyzed the interaction between IncRNA and protein using the STRING online software [13] and the combined score $>0.4$ was used as the cutoff criterion [14]. The PPI network was built using Cytoscape software [15].

\section{Results}

\section{Identification of relapse-related mRNAs and IncRNAs in Hodgkin lymphoma}

In the present study, we analyzed the publicly available gene expression dataset GSE17920 to identify relapse-related mRNAs and IncRNAs in Hodgkin lymphoma. A total of 9 early relapse $\mathrm{HL}$ and 24 late relapse $\mathrm{HL}$ samples were included in GSE17920. We firstly analyzed differentially expressed mRNAs in the GSE17920 database and found that 130 genes were up-regulated and 1209 genes were down-regulated in late relapse $\mathrm{HL}$ compared with early relapse $\mathrm{HL}$ (Figure $1 \mathrm{~A}$ ).

We compared the IncRNA expression patterns between early relapse $\mathrm{HL}$ and late relapse $\mathrm{HL}$. A total of 18 IncRNAs were dysregulated in late relapse $H L$ compared with early relapse $H L$ (Figure $1 \mathrm{~A}$ ). Among them, GGTA1P, PCBP1-AS1, GK3P and IL1ORB-AS1 were down-regulated and PGM5AS1, HCG18, CHRM3-AS2, PSMD6-AS2, SNHG6, LOC102606465, LOC100190986, GAS5, MIR29B2, PRKCQ-AS1, ITGB2-AS1, MIR142, LOC101060091 and LINC00926 were up-regulated in late relapse $\mathrm{HL}$ samples (Figure $1 \mathrm{~B}$ ).

\section{Construction of a relapse-related IncRNA- mRNA co-expression network}

Furthermore, we constructed a relapse-related IncRNA-mRNA co-expression network by calculating the Pearson correlation coefficient of the IncRNA-mRNA pair. In this study, only the IncRNA-mRNA pair with $|R|>0.65$ was selected for network construction. Our results revealed that the relapse-related IncRNA-mRNA co-expression network consisted of 18 InCRNAs and 762 DEGs (Figure 2). HCG18, PGM5-AS1, PSMD6-AS2, and SNHG6 acted as key IncRNAs in this network by affecting more than 200 mRNAs.

\section{GO and KEGG analysis of differentially expressed IncRNAs}

To obtain the potential functions of differentially expressed IncRNAs, we used GO and pathway analysis. The results of GO analysis showed that the upregulated genes were mainly involved in regulating responses to organic substances, immune system processes, responses to stress, cellular responses to organic substances, regulation of immune system processes, responses to chemicals, responses to stimuli, cellular responses to chemical stimuli, regulation of responses to stimuli, cell surface receptor signaling pathways, and cell activation (Figure $3 \mathrm{~A}$ ). Meanwhile, we found that the molecular functions of upregulated genes were mainly involved in protein binding, enzyme binding, carbohydrate derivative binding, 
A

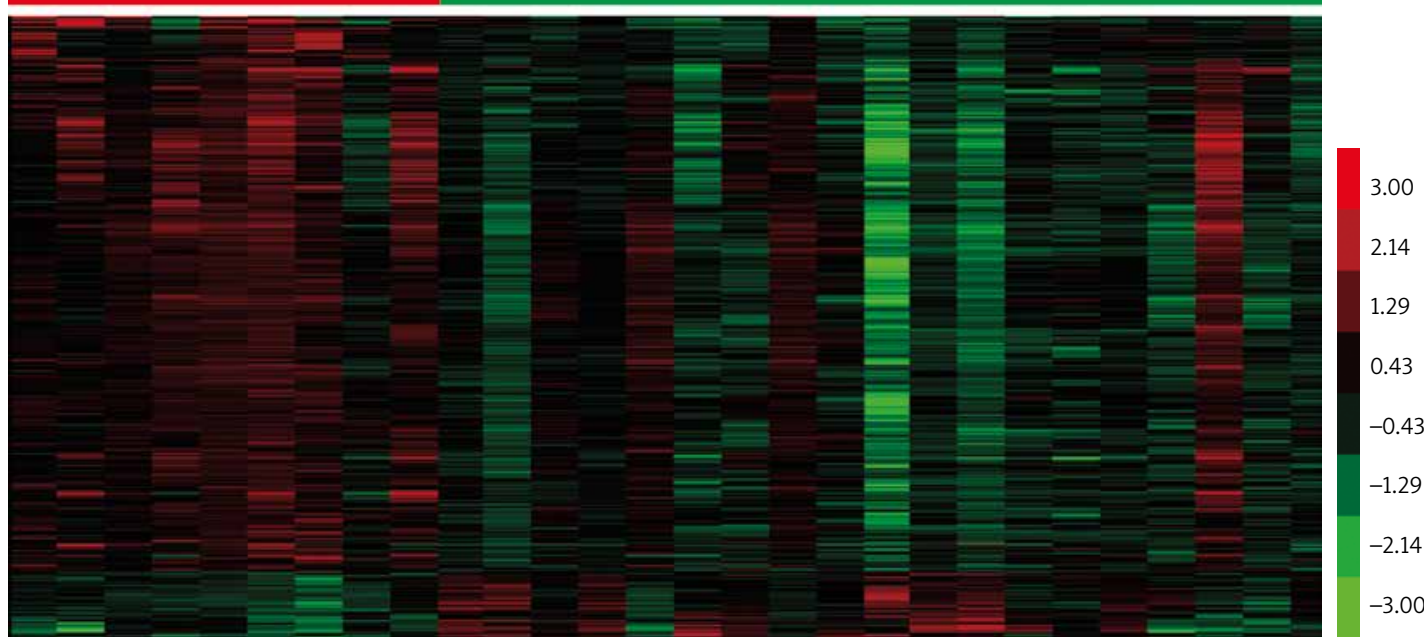

B
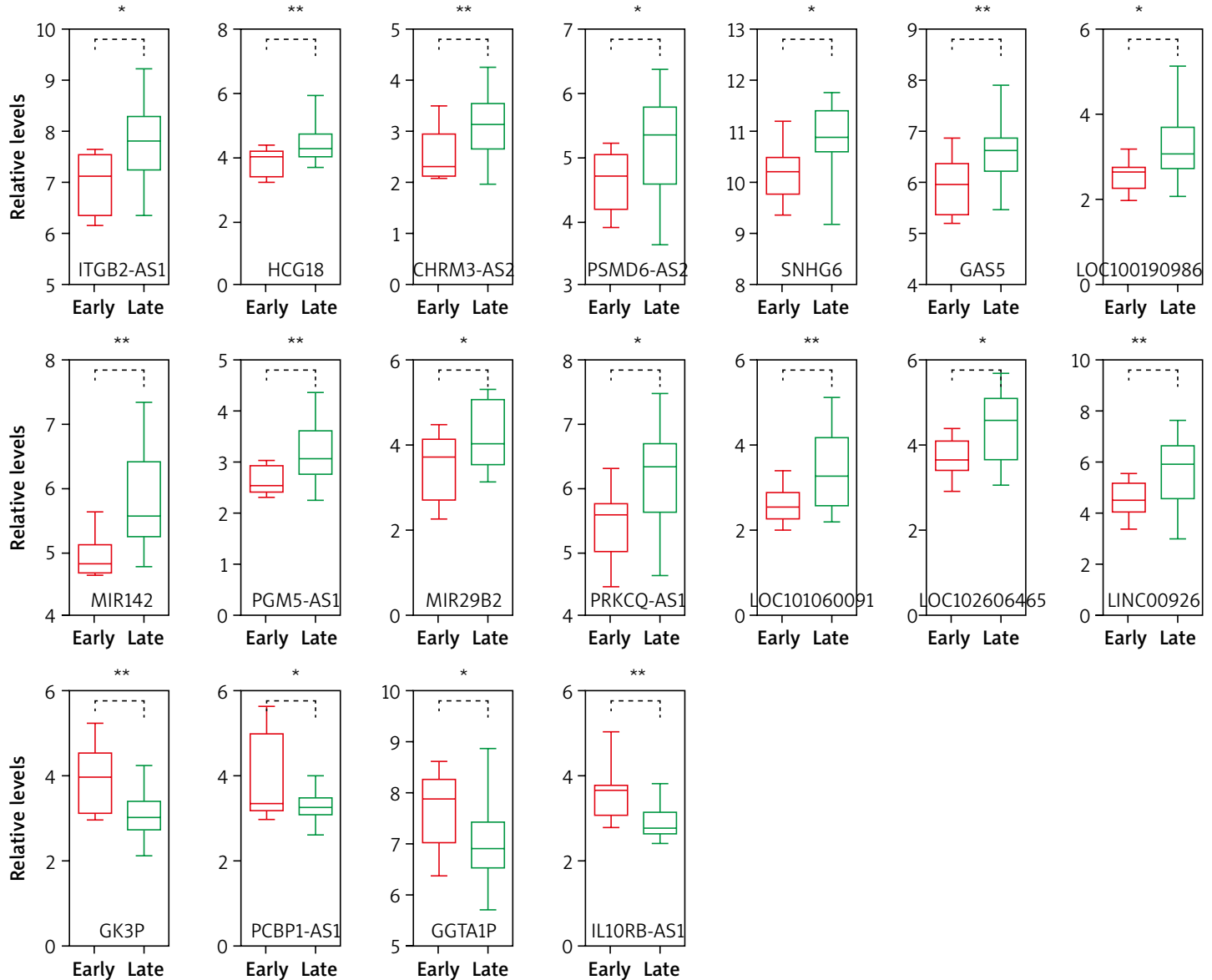

Figure 1. Identification of relapse-related mRNAs and IncRNAs in Hodgkin lymphoma. A - Hierarchical clustering analysis showed differential mRNAs and IncRNA expression in the HL by using GSE17920. B - GSE17920 analysis showed that 18 IncRNAs were dysregulated in late relapse $\mathrm{HL}$ compared with late relapse $\mathrm{HL}$ 


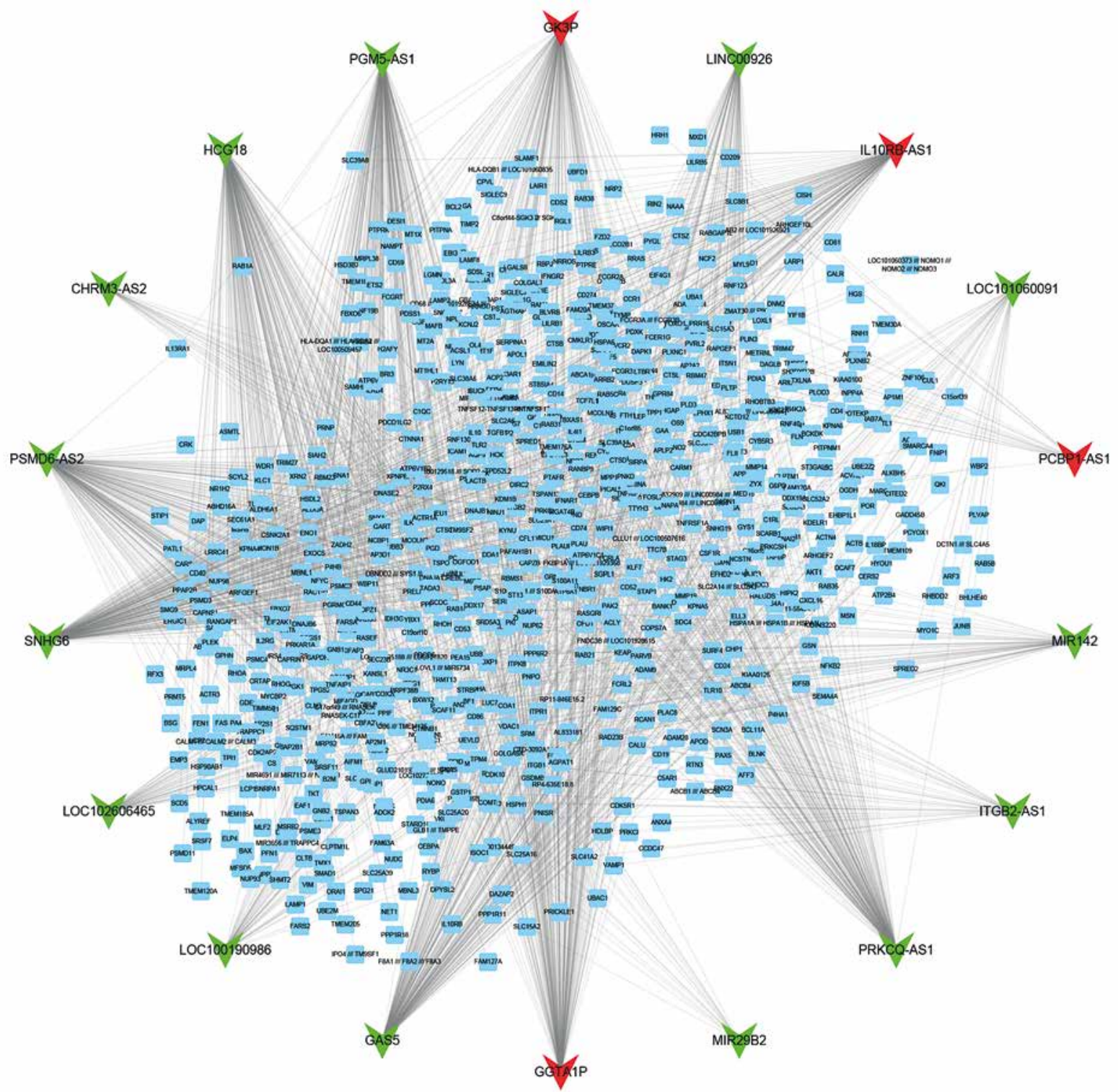

Figure 2. Construction of relapse-related IncRNA-mRNA co-expression networks in $\mathrm{HL}$. The co-expression networks showed a relapse-related IncRNA-mRNA co-expression network consisting of 18 IncRNAs and 762 DEGs

ion binding, glycoprotein binding, identical protein binding, monosaccharide binding, antigen binding and small molecule binding (Figure $3 \mathrm{~B}$ ).

The results of KEGG pathway analysis demonstrated that upregulated IncRNAs were mainly associated with the phagosome, lysosome, osteoclast differentiation, protein processing in endoplasmic reticulum, tuberculosis, Staphylococcus aureus infection, antigen processing and presentation, and carbon metabolism (Figure $3 \mathrm{C}$ ).

\section{Construction of relapse-related IncRNA- mediated PPI networks}

We next constructed relapse-related IncRNA-mediated PPI networks based on the information in the STRING database. Furthermore, the Mcode plugin was further used to identify key modules (cut-off $\geq 3$ and the nodes with edges $\geq 3$-core). In total, 17 hub-networks were identified in this study. The top 3 hub-networks with more than 20 nodes are shown in Figure 4. Hub-network 1 contained 53 nodes and 458 edges (Figure $3 \mathrm{D}$ ), hub-network 2 contained 37 nodes and 188 edges (Figure $3 \mathrm{E}$ ), and hub-network 3 had 39 nodes and 135 edges (Figure $3 \mathrm{~F}$ ).

\section{Construction of relapse-related IncRNA-mediated ceRNA networks}

We also constructed relapse-related IncRNA-mediated ceRNA to study the potential mechanism of IncRNAs regulating mRNA expression. We selected positively correlated IncRNA-mRNA pairs with $|R|>0.65$ to build ceRNA networks. Then, we predicted the potential interactions of miRNA- 
A

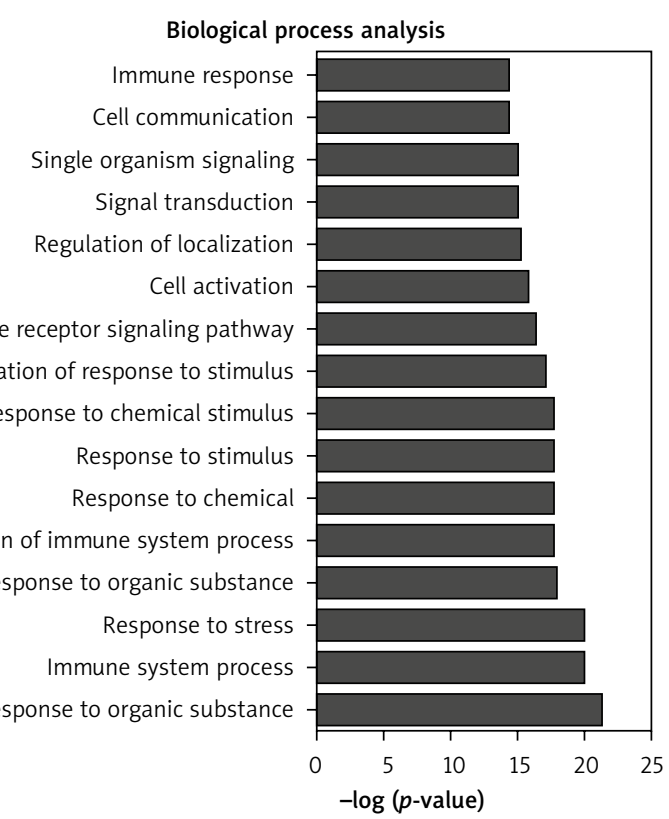

C

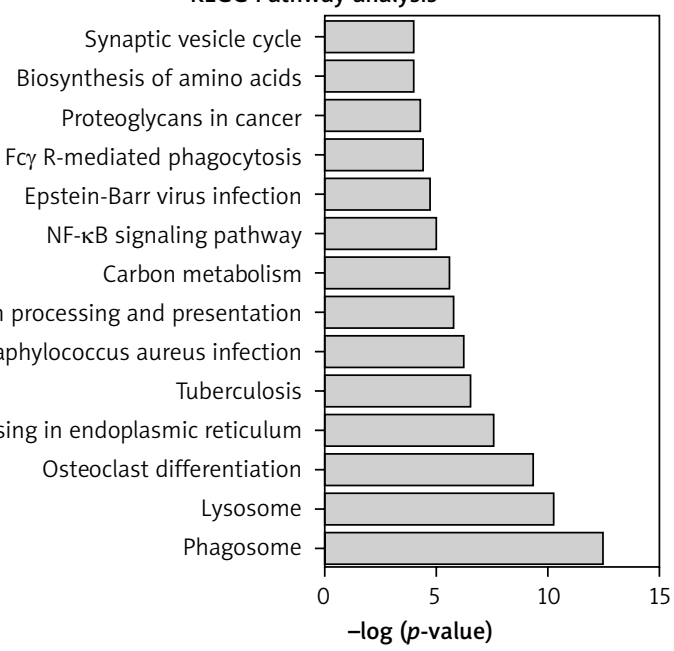

$E$

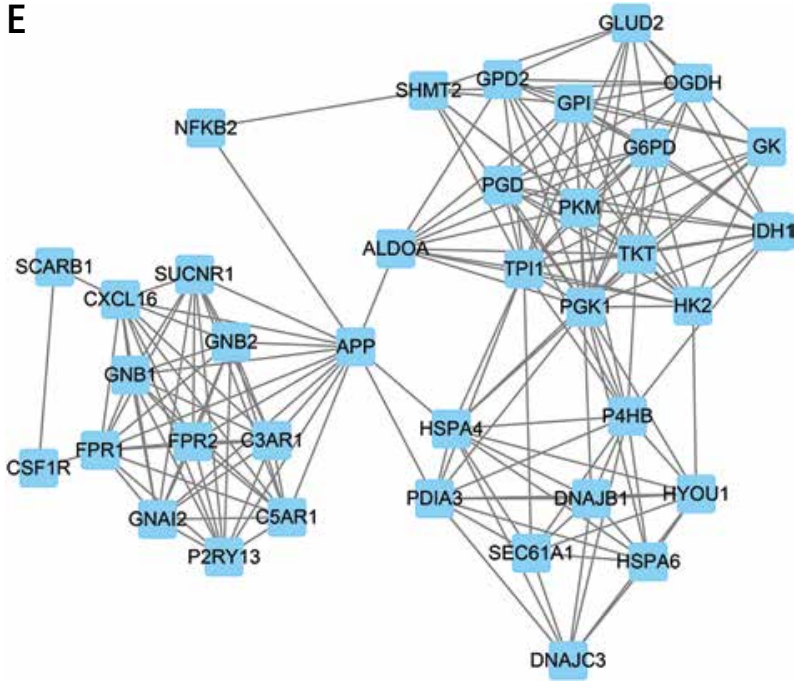

B
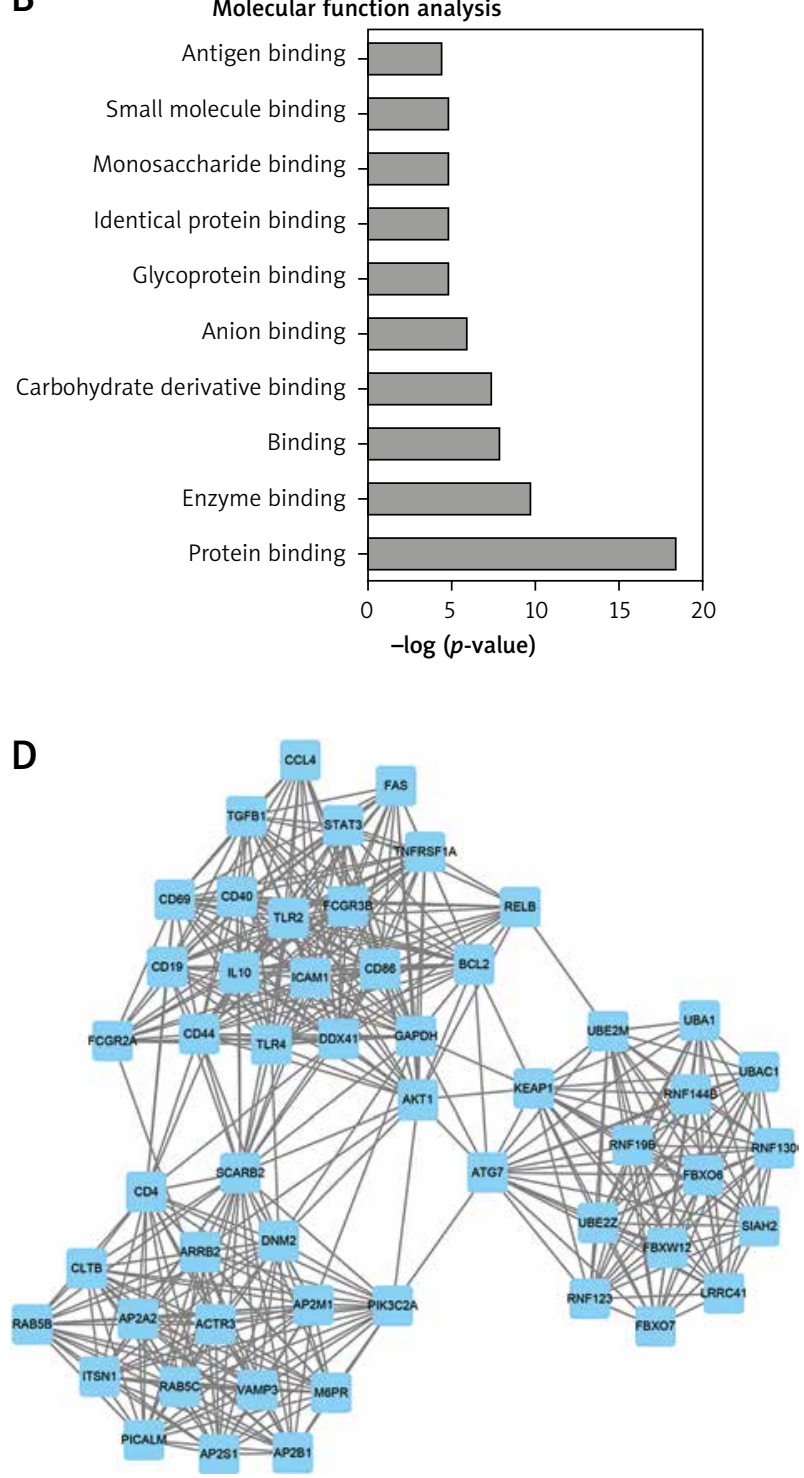

$\mathrm{F}$

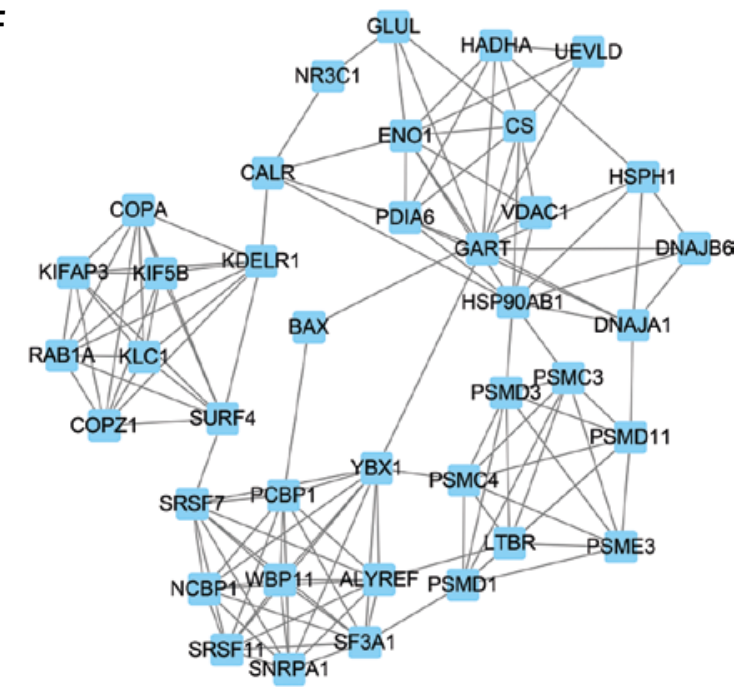

Figure 3. GO and KEGG analysis of differentially expressed IncRNAs in HL. (A-B) GO analysis shows differentially expressed IncRNA-related biological process analysis (A) and molecular function analysis (B). C - KEGG pathway analysis reveals differentially expressed IncRNA-related KEGG pathway analysis. D - Hub-network 1 contained 53 nodes and 458 edges. $\mathbf{E}$ - Hub-network 2 contained 37 nodes and 188 edges. F - Hub-network 3 contained 39 nodes and 135 edges 


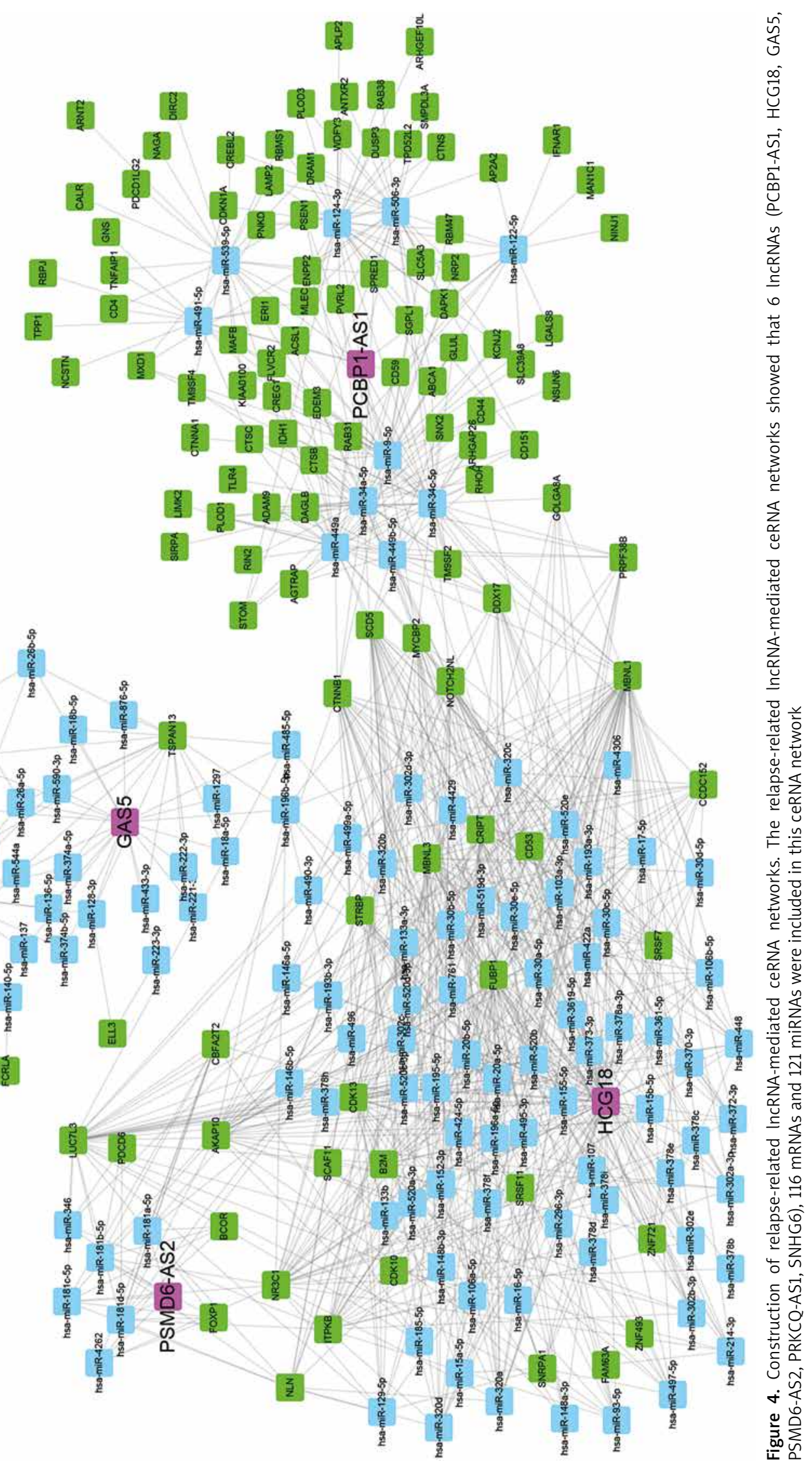


IncRNA and miRNA-mRNA using StarBase. As shown in Figure 4, 6 IncRNAs (PCBP1-AS1, HCG18, GAS5, PSMD6-AS2, PRKCQ-AS1, SNHG6), 116 mRNAs and 121 miRNAs were included in this ceRNA network. Interestingly, we found that PCBP1-AS1 and HCG18 were the key regulators in this network. According to our analysis, we found that HCG18 could sponge more than 75 miRNAs in the $\mathrm{HL}$ relapse-related progression and PCBP1-AS1 could regulate more than 60 mRNAs by competitive binding to hsa-miR-34a-5p, hsa-miR-9-5p, hsa-miR-34c-5p, hsa-miR-539-5p, hsa-miR-122-5p, hsa-miR-124-3p, hsa-miR-449a, hsa-miR-449b-5p, hsa-miR-491-5p, and hsa-miR-506-3p.

\section{Discussion}

Hodgkin lymphoma $(\mathrm{HL})$ is the main common lymphoma throughout the western countries [16]. Relapse of Hodgkin lymphoma after treatment is a major therapeutic challenge. However, the molecular mechanisms involved in the relapse of $\mathrm{HL}$ remained unclear. Emerging studies have shown that IncRNAs play key roles in cancer progres sion. In $\mathrm{HL}$, only two reports have indicated that IncRNAs were involved in $\mathrm{HL}$ progression. For example, Tayari et al. identified HL-specific IncRNAs by using microarray profiling [10]. In this study, we analyzed relapse-related IncRNAs in HL using the public dataset GSE17920. A total of 18 IncRNAs were dysregulated in the relapse progression of HL. Among them, GGTA1P, PCBP1-AS1, GK3P and IL10RB-AS1 were down-regulated and PGM5AS1, HCG18, CHRM3-AS2, PSMD6-AS2, SNHG6, LOC102606465, LOC100190986, GAS5, MIR29B2, PRKCQ-AS1, ITGB2-AS1, MIR142, LOC101060091 and LINC00926 were up-regulated in late-relapse $\mathrm{HL}$ samples compare to early-relapse HL. To the best of our knowledge, this is the first study to reveal relapse-related IncRNAs in $\mathrm{HL}$.

Among these IncRNAs, SNHG6 and GAS5 have been reported to be key regulators in cancers. SNHG6 could promote esophageal squamous cell carcinoma, glioma, gastric cancer and hepatocellular carcinoma cell proliferation though ceRNA mechanisms [17-20]. GAS5 acted as a tumor suppressor involved in various cancers, including breast cancer, lung cancer, liver cancer and gastric cancer [21-24]. However, the molecular roles of these IncRNAs in $\mathrm{HL}$ remained unknown. In the present study, we first constructed relapse-related IncRNA-mRNA co-expression networks which included 18 IncRNAs and 762 mRNAs. Moreover, we performed bioinformatics analysis. Our results showed these IncRNAs were mainly involved in regulating responses to organic substances, immune system processes, responses to stress, cellular responses to organic substances, regulation of immune system processes, and phagosome and lysosome-related pathways.
Finally, we constructed relapse-related IncRNAs mediated PPI networks. These results strongly suggested that IncRNAs also play important roles in relapse progression of $\mathrm{HL}$.

The competitive endogenous RNA (ceRNA) hypothesis showed that IncRNAs could competitively bind to miRNAs to regulate protein-coding gene expression [25]. Emerging studies have shown that ceRNA play crucial roles in the development and progression of human diseases, such as prostate cancer, gliomas, liver cancer, and diabetes [26]. The ceRNA hypothesis provides an important mechanism to explain how IncRNAs regulate disease progression. Here, we constructed relapse-related IncRNA-mediated ceRNA to explore the potential mechanism of IncRNAs regulating mRNA expression. A total of 6 IncRNAs (PCBP1-AS1, HCG18, GAS5, PSMD6-AS2, PRKCO-AS1, SNHG6), 116 mRNAs and 121 miRNAs were included in this ceRNA network. Interestingly, we found that PCBP1-AS1 and HCG18 were the key regulators in this network. According to our analysis, we found that HCG18 could sponge more than 75 miRNAs and PCBP1-AS1 could regulate more than 60 mRNAs. PCBP1-AS1 was a novel IncRNA involved in oral squamous cell carcinoma progression [27] and IncRNA HCG18 promoted intervertebral disc degeneration by sponging miR-146a-5p and regulating TRAF6 expression [28].

In conclusion, we identified a total of 18 lncRNAs that were found to be dysregulated between early relapse and late relapse HL. Furthermore, we constructed co-expression networks for these relapse-related IncRNAs. Bioinformatics analysis revealed that these IncRNAs were significantly associated with immune system processes, responses to stress, and responses to chemicals. Moreover, we constructed a relapse-related IncRNA-miRNA-mRNA network to explore the potential mechanism of these IncRNAs. OF note, HCG18 and PCBP1-AS1 were identified as key IncRNAs in $\mathrm{HL}$ relapse. Although the results must be confirmed in a prospective clinical trial on a larger group of patients, we trust that this work will give a potential new therapeutic and prognostic target for $\mathrm{HL}$.

\section{Acknowledgments}

Yuexiong Liang and Haifeng Zhu contributed equally to this work. Jing Chen, Wei Lin and Bing Li contributed equally to this work.

\section{Conflict of interest}

The authors declare no conflict of interest.

\section{References \\ 1. Agostinelli C, Pileri SJ. Pathobiology of Hodgkin lympho- ma. Mediterr J Hematol Infect Dis 2014; 6: e2014040.}


2. Engert A, Eichenauer D, Dreyling M; Group EGW. Hodgkin's lymphoma: ESMO clinical recommendations for diagnosis, treatment and follow-up. Ann Oncol 2009; 20: iv108-9.

3. Biasoli I, Stamatoullas A, Meignin V, et al. Nodular, lymphocyte-predominant Hodgkin lymphoma. Cancer 2010; 116: 631-9.

4. Aleman BM, van den Belt-Dusebout AW, Klokman WJ, van't Veer MB, Bartelink H, van Leeuwen FE. Long-term cause-specific mortality of patients treated for Hodgkin's disease. J Clin Oncol 2003; 21: 3431-9.

5. Zamore PD, Haley B. Ribo-gnome: the big world of small RNAs. Science 2005; 309: 1519-24.

6. Kornienko AE, Guenzl PM, Barlow DP, Pauler FM. Gene regulation by the act of long non-coding RNA transcription. BMC Biol 2013; 11: 59.

7. Wan X, Huang W, Yang S, et al. Identification of androgen-responsive IncRNAs as diagnostic and prognostic markers for prostate cancer. Oncotarget 2016; 7: 60503-18.

8. Wang Z, Yang B, Zhang M, et al. IncRNA epigenetic landscape analysis identifies EPIC1 as an olncogenic IncRNA that Interacts with MYC and promotes cell-cycle progression in cancer. Cancer Cell 2018; 33: 706-20.e9.

9. Biswas S, Thomas AA, Chen S, et al. MALAT1: an epigenetic regulator of inflammation in diabetic retinopathy. Sci Rep 2018; 8: 6526.

10. Tayari MM, Winkle M, Kortman G, et al. Long noncoding RNA expression profiling in normal B-cell subsets and Hodgkin lymphoma reveals Hodgkin and Reed-Sternberg cell-specific long noncoding RNAs. Am J Pathol 2016; 186: 2462-72.

11. Leucci E, Patella F, Waage J, et al. microRNA-9 targets the long non-coding RNA MALAT1 for degradation in the nucleus. Sci Rep 2013; 3: 2535.

12. Zhang X, Sun S, Pu JK, et al. Long non-coding RNA expression profiles predict clinical phenotypes in glioma. Neurobiol Dis 2012; 48: 1-8.

13. Zhang D, Ma W, He Y, et al. Data of the interacting protein networks and nucleotide metabolism pathways related to NDK and NT5. Data Brief 2016; 9: 1063-6.

14. Yan H, Li Z, Shen $\mathrm{Q}$ et al. Aberrant expression of cell cycle and material metabolism related genes contributes to hepatocellular carcinoma occurrence. Pathol Res Pract 2017; 213: 316-21.

15. Kohl M, Wiese S, Warscheid B. Cytoscape: software for visualization and analysis of biological networks. Methods Mol Biol 2011; 696: 291-303.

16. Siegel RL, Miller KD, Jemal A. Cancer statistics, 2015. CA Cancer J Clin 2015; 65: 5-29.

17. Ruan J, Zheng L, Hu N, et al. Long noncoding RNA SNHG6 promotes osteosarcoma cell proliferation through regulating p21 and KLF2. Arch Biochem Biophys 2018; 646: 128-36.

18. Cai G, Zhu Q, Yuan L, Lan Q. LncRNA SNHG6 acts as a prognostic factor to regulate cell proliferation in glioma through targeting p21. Biomed Pharmacother 2018; 102: 452-7.

19. Yan K, Tian J, Shi W, Xia H, Zhu Y. LncRNA SNHG6 is associated with poor prognosis of gastric cancer and promotes cell proliferation and EMT through epigenetically silencing p27 and sponging miR-101-3p. Cell Physiol Biochemy 2017; 42: 999-1012.

20. Cao C, Zhang T, Zhang D, et al. The long non-coding RNA, SNHG6-003, functions as a competing endogenous RNA to promote the progression of hepatocellular carcinoma. Oncogene 2017; 36: 1112-22.
21. Mourtada-Maarabouni M, Pickard M, Hedge V, Farzaneh F, Williams G. GAS5, a non-protein-coding RNA, controls apoptosis and is downregulated in breast cancer. Oncogene 2009; 28: 195-208.

22. Wu Y, Lyu H, Liu H, Shi X, Song Y, Liu B. Downregulation of the long noncoding RNA GAS5-AS1 contributes to tumor metastasis in non-small cell lung cancer. Sci Rep 2016; 6: 31093.

23. Tu ZQ, Li RJ, Mei JZ, Li XH. Down-regulation of long non-coding RNA GAS5 is associated with the prognosis of hepatocellular carcinoma. Int J Clin Exp Pathol 2014; 7: 4303-9.

24. Sun M, Jin FY, Xia R, et al. Decreased expression of long noncoding RNA GAS5 indicates a poor prognosis and promotes cell proliferation in gastric cancer. BMC Cancer 2014; 14: 319.

25. Thomson DW, Dinger ME. Endogenous microRNA sponges: evidence and controversy. Nat Rev Genet 2016; 17: 272-83.

26. Van Roosbroeck K, Pollet J, Calin GA. miRNAs and long noncoding RNAs as biomarkers in human diseases. Exp Rev Mol Diagn 2013; 13: 183-204.

27. Feng L, Houck JR, Lohavanichbutr P, Chen C. Transcriptome analysis reveals differentially expressed IncRNAs between oral squamous cell carcinoma and healthy oral mucosa. Oncotarget 2017; 8: 31521-31.

28. Xi Y, Jiang T, Wang $W$, et al. Long non-coding HCG18 promotes intervertebral disc degeneration by sponging miR-146a-5p and regulating TRAF6 expression. Sci Rep 2017; 7: 13234. 